\title{
Observer Based Adaptive Output Feedback Tracking Control of Robot Manipulators
}

\author{
Erkan Zergeroglu, Enver Tatlicioglu
}

\begin{abstract}
In this paper, we propose an observer based adaptive output feedback (OFB) tracking controller for rigidlink robot manipulators. Specifically, we used a model independent observer in conjuction with a desired compensation adaptation law (DCAL) to remove the link velocity dependency of the controller and achieved asymptotic stability of the observer-controller couple despite the uncertainties associated with the system dynamics. Lyapunov based arguments are utilized to illustrate the stability of the proposed controller. Simulation results are included to demonstrate the performance of observer-controller couple.
\end{abstract}

\section{INTRODUCTION}

Controlling robot manipulators using only link position measurements has received considerable attention due to the fact that nearly all commercially available robot manipulators do not have link velocity sensors and the ones that have velocity sensors the sensor outputs are, most of the time, contaminated with noise. The existing solutions to the forementioned problem can be categorized as observer based [1], [2] and filter based [3], [4], [5], [6], [7] methods. In most observer based methods, a model based observer [2], [8] is used to estimate the velocity signal, where in filtered based approaches surrogate filters are used to overcome the need of velocity measurements. However, when the robot parameters are not precisely known, the observer based methods fail as most of them require the exact knowledge of system parameters.

In this paper, we present a new model-free observer based adaptive output feedback tracking controller for robot manipulators. Inspired by the work of [9], the proposed controller utilizes a new model-free observer structure, in conjunction with a DCAL based adaptation formulation and achieves semi-global asymptotic tracking performance despite the lack of link velocity measurements and parametric uncertainties in the robot dynamics. Compared to the model-free observers in the literature (as in [10]) the proposed method has the advantage of compensating for the uncertainties in the system dynamics.

The rest of the paper is organized as follows. In Section II, the dynamic model of the robot manipulator and model properties that are used in the analysis and design of the proposed observer-controller couple are presented, while, Section III

E. Zergeroglu is with the Department of Computer Engineering, Gebze Institute of Technology, 41400, Gebze, Kocaeli, Turkey.

ezerger@bilmuh.gyte.edu.tr

E. Tatlicioglu is with the Department of Electrical \& Electronics Engineering, Izmir Institute of Technology, Gulbahce Koyu, Urla, Izmir, 35430 Turkey.

envertatlicioglueiyte.edu.tr contains error system development and problem formulation. In Section IV, design and stability analysis of the controllerobserver couple are proposed. In Section V, we demonstrate the effectiveness of the proposed method through simulation results obtained from a two link, direct drive planar robot manipulator. Concluding remarks are presented in Section VI.

\section{RoBOt MODEL}

The mathematical model for an $n$ DOF, revolute joint, direct drive, robot manipulator is assumed to have the following form [11]

$$
M(q) \ddot{q}+V_{m}(q, \dot{q}) \dot{q}+G(q)+F_{d} \dot{q}=\tau
$$

where $q(t), \dot{q}(t), \ddot{q}(t) \in \mathbb{R}^{n}$ denote the link position, velocity, and acceleration, respectively, $M(q) \in \mathbb{R}^{n \times n}$ represents the positive-definite, symmetric inertia matrix, $V_{m}(q, \dot{q}) \in \mathbb{R}^{n \times n}$ represents the centripetal-Coriolis matrix, $G(q) \in \mathbb{R}^{n}$ is the gravitational vector, $F_{d} \in \mathbb{R}^{n \times n}$ denotes the constant, diagonal, positive-definite, viscous friction matrix, and $\tau(t) \in \mathbb{R}^{n}$ represents the torque input control vector. We will assume that the left-hand side of (1) is first-order differentiable.

The dynamic system given by (1) exhibits the following properties that are utilized in the subsequent control development and stability analysis.

Property 1: The inertia matrix can be bounded by the following inequalities [11]

$$
m_{1} I_{n} \leq M(q) \leq m_{2} I_{n}
$$

where $m_{1}$ and $m_{2}$ are positive constants, and $I_{n}$ is the $n \times n$ identity matrix. Likewise the inverse of the inertia matrix can be bounded as follows

$$
\frac{1}{m_{2}} I_{n} \leq M^{-1}(q) \leq \frac{1}{m_{1}} I_{n} .
$$

Property 2: The inertia and the centripetal-Coriolis matrices satisfy the following relationship [12]

$$
\xi^{T}\left(\frac{1}{2} \dot{M}(q)-V_{m}(q, \dot{q})\right) \xi=0 \quad \forall \xi \in \mathbb{R}^{n} .
$$

Property 3: The centripetal-Coriolis matrix satisfies the following relationship [8]

$$
V_{m}(q, \nu) \xi=V_{m}(q, \xi) \nu \quad \forall \xi, \nu \in \mathbb{R}^{n} .
$$

Property 4: The norm of the centripetal-Coriolis and friction matrices can be upper bounded as follows [11]

$$
\left\|V_{m}(q, \xi)\right\| \leq \zeta_{c 1}\|\xi\|, \quad\left\|F_{d}\right\| \leq \zeta_{f} \quad \forall \xi \in \mathbb{R}^{n}
$$

where $\zeta_{c 1}, \zeta_{f} \in \mathbb{R}$ are positive bounding constants. 
Property 5: The robot dynamics given in (1) can be linearly parameterized as follows [11]

$$
Y(q, \dot{q}, \ddot{q}) \theta=M(q) \ddot{q}+V_{m}(q, \dot{q}) \dot{q}+G(q)+F_{d} \dot{q}
$$

where $\theta \in \mathbb{R}^{p}$ contains the constant system parameters, and $Y(q, \dot{q}, \ddot{q}) \in \mathbb{R}^{n \times p}$ denotes the regression matrix which is a function of $q(t), \dot{q}(t)$, and $\ddot{q}(t)$. The formulation of (7) can also written in terms of the desired trajectory in the following manner

$$
Y_{d}\left(q_{d}, \dot{q}_{d}, \ddot{q}_{d}\right) \theta=M\left(q_{d}\right) \ddot{q}_{d}+V_{m}\left(q_{d}, \dot{q}_{d}\right) \dot{q}_{d}+G\left(q_{d}\right)+F_{d} \dot{q}_{d}
$$

where the desired regression matrix $Y_{d}\left(q_{d}, \dot{q}_{d}, \ddot{q}_{d}\right) \in \mathbb{R}^{n \times p}$ is a function of the desired link position, velocity, and acceleration, denoted by $q_{d}(t), \dot{q}_{d}(t), \ddot{q}_{d}(t) \in \mathbb{R}^{n}$, respectively.

Property 6: The inertia, centripetal-Coriolis, and gravity terms of (1) can be upper bounded as follows [13]

$$
\begin{gathered}
\|M(\xi)-M(\nu)\|_{i \infty} \leq \zeta_{m 1}\|(\xi-\nu)\| \\
\left\|M^{-1}(\xi)-M^{-1}(\nu)\right\|_{i \infty} \leq \zeta_{m 2}\|(\xi-\nu)\| \\
\left\|V_{m}(\xi, w)-V_{m}(\nu, w)\right\|_{i \infty} \leq \zeta_{c 2}\|w\|\|(\xi-\nu)\| \\
\|G(\xi)-G(\nu)\| \leq \zeta_{g}\|(\xi-\nu)\|
\end{gathered}
$$

$\forall \xi, \nu, w \in \mathbb{R}^{n}, \zeta_{m 1}, \zeta_{m 2}, \zeta_{c 2}, \zeta_{g}$ are positive bounding constants, and $\|\cdot\|_{i \infty}$ denotes the induced norm of a matrix.

\section{Problem Formulation}

The control objective is to design a link position tracking controller for the robot manipulator model given by (1) under the constraints that only the link position variable $q(t)$ is available for measurement and that the parameter vector $\theta$ introduced in (7) is unknown. We will quantify the control objective by defining the link position tracking error, denoted by $e(t) \in \mathbb{R}^{n}$, as follows

$$
e \triangleq q_{d}-q
$$

where we assume that $q_{d}(t)$ and its first three time derivatives are bounded functions of time. To account for the unmeasurable link velocity constraint, we define $\dot{\hat{q}}(t) \in \mathbb{R}^{n}$ as the observed velocity signal. The corresponding velocity and position observation error signals, denoted by $\dot{\tilde{q}}(t), \tilde{q}(t) \in$ $\mathbb{R}^{n}$, respectively, are defined as

$$
\begin{aligned}
& \dot{\tilde{q}}=\dot{q}-\dot{\hat{q}}, \\
& \tilde{q}=q-\hat{q} .
\end{aligned}
$$

To ease the presentation of the subsequent analysis, we will use two auxiliary variables, filtered tracking error, denoted by $r(t) \in \mathbb{R}^{n}$, and filtered observation error, denoted by $s(t) \in \mathbb{R}^{n}$, as

$$
r \triangleq \dot{e}+\alpha e, \text { and } s \triangleq \dot{\tilde{q}}+\alpha \tilde{q}
$$

where $\alpha \in \mathbb{R}$ is a positive control gain. It should be noted that, regulating $r(t)$ and $s(t)$ ensures the regulation of $e(t)$ and $\tilde{q}(t)$, respectively. In addition, we define the difference between the actual and estimated parameters as follows

$$
\tilde{\theta} \triangleq \theta-\hat{\theta}
$$

where $\tilde{\theta}(t) \in \mathbb{R}^{p}$ represents the parameter estimation error vector, and $\hat{\theta}(t) \in \mathbb{R}^{p}$ represents a dynamic estimate of $\theta$.

\section{OBSERVER-CONTROLlER DESIGN}

Based on the subsequent error system development and the stability analysis, we propose the following velocity observer

$$
\begin{aligned}
& \dot{\hat{q}}=p+K_{0} \tilde{q}-K_{c} e \\
& \dot{p}=K_{1} \operatorname{Sgn}(\tilde{q})+K_{2} \tilde{q}-\alpha K_{c} e
\end{aligned}
$$

where $p(t) \in \mathbb{R}^{n}$ is an auxiliary variable, $K_{0}, K_{c}, K_{1}$, $K_{2} \in \mathbb{R}^{n \times n}$ are diagonal, positive define gain matrices, and $\operatorname{Sgn}(\cdot) \in \mathbb{R}^{n}$ is defined as

$\operatorname{Sgn}(\zeta)=\left[\begin{array}{lll}\operatorname{sgn}\left(\zeta_{1}\right) & \operatorname{sgn}\left(\zeta_{2}\right) & \cdots \operatorname{sgn}\left(\zeta_{n}\right)\end{array}\right]^{T} \forall \zeta \in \mathbb{R}^{n}$

with $\operatorname{sgn}(\cdot)$ being the scalar signum function. It is straightforward to show that the time derivative of (14) yields

$$
\ddot{\hat{q}}=K_{1} \operatorname{Sgn}(\tilde{q})+K_{2} \tilde{q}+K_{0} \dot{\tilde{q}}-K_{c} r
$$

where the definition of $r(t)$ given in (12) has been utilized. Based on the subsequent stability analysis, the control input torque, $\tau(t)$, is designed as

$$
\tau=Y_{d} \hat{\theta}+K_{p} e+K_{c} \alpha\left(q_{d}-\hat{q}\right)+K_{c}\left(\dot{q}_{d}-\dot{\hat{q}}\right)
$$

where $K_{p} \in \mathbb{R}^{n \times n}$ is a diagonal positive define control gain matrix and the parameter estimate vector $\hat{\theta}(t)$ is generated according to the following update rule

$$
\begin{aligned}
\hat{\theta}= & \operatorname{Proj}\left\{\Gamma \left(Y_{d}^{T} e-\int_{0}^{t} \frac{d}{d \sigma}\left\{Y_{d}^{T}(\sigma)\right\} e(\sigma) d \sigma\right.\right. \\
& \left.\left.+\alpha \int_{0}^{t} Y_{d}^{T}(\sigma) e(\sigma) d \sigma\right)\right\}
\end{aligned}
$$

with $\Gamma \in \mathbb{R}^{p \times p}$ being a constant, diagonal, positive-definite, adaptation gain matrix and $\operatorname{Proj}\{\cdot\}$ is a projection operator introduced to ensure the boundedness of $\hat{\theta}(t)$ and its time derivative.

At this point, we want to note that, it is clear that, from the observer-controller couple in (14) and (17), and the parameter estimate law in (18), the proposed methodology can be implemented without link velocity measurements. However, for the ease of presentetation, we will make use of the fact that

$$
q_{d}-\hat{q}=e+\tilde{q}
$$

to re-arrange (17) in the following advantageous form

$$
\tau=Y_{d} \hat{\theta}+K_{p} e+K_{c} r+K_{c} s
$$

Similarly, after taking the time derivative of (18), the parameter update law can be re-written in the following form

$$
\dot{\hat{\theta}}=\operatorname{Proj}\left\{\Gamma Y_{d}^{T} r\right\}
$$

In the rest of paper, we will make use of these new definitions for the controller and the parameter estimation law. 


\section{A. Observer Analysis}

After utilizing (1) for the link acceleration signal and (16) for the time derivative of the observed velocity signal, the observation error dynamics can be obtained as

$$
\begin{aligned}
\ddot{\tilde{q}} & =\ddot{q}-\ddot{\hat{q}} \\
& =N_{0}-K_{1} \operatorname{Sgn}(\tilde{q})-K_{2} \tilde{q}-K_{0} \dot{\tilde{q}}+K_{c} r
\end{aligned}
$$

where the auxiliary term $N_{0}(t) \in \mathbb{R}^{n}$ is defined as

$$
N_{0}=M^{-1}(q)\left\{\tau-V_{m}(q, \dot{q}) \dot{q}-G(q)-F_{d} \dot{q}\right\} .
$$

After inserting (20) and (8) into (23), we can explicitly obtain the following expression

$$
N_{0}=N_{d}+N_{b}
$$

where the auxiliary variables $N_{d}(t) \in \mathbb{R}^{n}$ and $N_{b}(t) \in \mathbb{R}^{n}$ are specifically defined as

$$
N_{d}(t) \triangleq \ddot{q}_{d}+M^{-1}\left(q_{d}\right) Y_{d} \tilde{\theta}
$$

and

$$
\begin{aligned}
N_{b}(t) \triangleq & \left(M^{-1}(q)-M^{-1}\left(q_{d}\right)\right) M\left(q_{d}\right) \ddot{q}_{d} \\
& +\left(M^{-1}(q)-M^{-1}\left(q_{d}\right)\right) Y_{d} \tilde{\theta} \\
& +M^{-1}(q)\left\{V_{m}\left(q_{d}, \dot{q}_{d}\right) \dot{q}_{d}-V_{m}(q, \dot{q}) \dot{q}\right. \\
& +G\left(q_{d}\right)-G(q)+F_{d}\left(\dot{q}_{d}-\dot{q}\right) \\
& \left.+K_{p} e+K_{c}(r+s)\right\}
\end{aligned}
$$

Remark 1: After exploiting the boundedness properties of the desired trajectory, we can show that both $N_{d}(t)$ and its time derivative, denoted by $\dot{N}_{d}(t)$, are bounded signals. Furthermore, as illustrated in Appendix I, after using (5), (6), (9), and the mean value theorem [14], $N_{b}(t)$ can be upper bounded as

$$
\left\|N_{b}(t)\right\| \leq \rho_{o 1}\|e\|+\rho_{o 2}\|r\|+\rho_{o 3}\|r\|^{2}+\rho_{o 4}\|s\|
$$

where $\rho_{o i}, i=1, . ., 4$ are some positive known bounding functions that depend on the model parameters and the desired trajectory, and $\|\cdot\|$ denotes the standard Euclidean norm.

After taking the time derivative of $s(t)$ and inserting for (22), the dynamics for the filtered observation error $s(t)$ can be obtained as follows

$$
\dot{s}=N_{d}+N_{b}-K_{1} \operatorname{Sgn}(\tilde{q})-K_{2} \tilde{q}-\left(K_{0}-\alpha\right) \dot{\tilde{q}}+K_{c} r .
$$

Provided that the observer gains are selected to satisfy

$$
\alpha\left(K_{0}-\alpha\right)=K_{2}
$$

the expression in (28) can be rearranged to have the following form

$$
\dot{s}=N_{d}+N_{b}-K_{1} \operatorname{Sgn}(\tilde{q})-\frac{K_{2}}{\alpha} s+K_{c} r
$$

which enables us to state the following preliminary Lyapunov-like analysis for the observer. Specifically, we define the following non-negative scalar function $V_{0}(t) \in \mathbb{R}$

$$
V_{0}=\frac{1}{2} s^{T} s+P_{0}
$$

where the scalar auxiliary function $P_{0}(t) \in \mathbb{R}$ is defined as

$$
P_{0}=\zeta_{0}-\int_{t_{0}}^{t} w_{0}(\sigma) d \sigma
$$

where $w_{0}(t) \in \mathbb{R}$ and the non-negative constant $\zeta_{0} \in \mathbb{R}$ are defined as

$$
\begin{gathered}
w_{0} \triangleq s^{T}\left[N_{b}-K_{1} \operatorname{Sgn}(\tilde{q})\right] \\
\zeta_{0} \triangleq \sum_{i=0}^{n} K_{1 i}\left|\tilde{q}_{i}(0)\right|-\tilde{q}^{T}(0) N_{d}(0)
\end{gathered}
$$

where the subscript $i=1,2, \ldots, n$ denotes the $\mathrm{i}^{\text {th }}$ element of a vector or a diagonal matrix. Following a similar analysis to that of [9] and [15], it can be proven that when $K_{1}$ satisfies the following sufficient condition

$$
K_{1 i}>\left\|N_{d i}(t)\right\|_{\infty}+\frac{1}{\alpha}\left\|\dot{N}_{d i}(t)\right\|_{\infty}
$$

where $\|\cdot\|_{\infty}$ denotes the $\mathcal{L}_{\infty}$ norm, then $P_{0}(t)$ in (32) is always non-zero (i.e., $P_{0}(t) \geq 0$ ) and $V_{0}(t)$ is a positivedefinite Lyapunov function with respect to $s(t)$ and $\sqrt{P_{0}(t)}$. After taking the time derivative of (31), we obtain

$$
\dot{V}_{0}=s^{T}\left[-\frac{K_{2}}{\alpha} s+K_{c} r+N_{b}\right]
$$

where (30), the time derivative of (32), and (33) were utilized. The first term in the brackets in (35) will be used for both damping the unwanted effects of the term $N_{b}(t)$ in the composite stability analysis and to ensure the convergence of the observation error. The second term is designed to cancel out the interconnection term between the observer-controller subsystem. At this point, we are ready to proceed to the error system development.

\section{B. Error System Development}

To obtain the dynamics of $r(t)$, we take its time derivative and premultiply the resulting equation by $M(q)$, utilize (1) and (10) and perform some algebraic manipulation, to obtain

$$
M(q) \dot{r}=-V_{m}(q, \dot{q}) r+Y_{s} \theta-\tau
$$

where the auxiliary term $Y_{s}(t) \theta \in \mathbb{R}^{n}$ is defined as

$Y_{s} \theta=M(q)\left(\ddot{q}_{d}+\alpha \dot{e}\right)+V_{m}(q, \dot{q})\left(\dot{q}_{d}+\alpha e\right)+G(q)+F_{d} \dot{q}$.

After substituting the control law in (20) into (36), we obtain the following closed-loop dynamics for $r(t)$

$$
M(q) \dot{r}=-V_{m}(q, \dot{q}) r+\chi-K_{c} r-K_{c} s-K_{p} e
$$

where the disturbance-like term $\chi(r, e, t) \in \mathbb{R}^{n}$ is defined as follows

$$
\chi=Y_{s} \theta-Y_{d} \theta
$$

with $Y_{d}(t) \theta$ being defined in (8).

Remark 2: As illustrated in [11], and also shown in Appendix I, we can exploit the boundedness properties of the desired trajectory, and Properties 3, 4, and 6, to show that the norm of $\chi(\cdot)$ can be upper bounded as

$$
\|\chi\| \leq \rho_{1}(e)\|e\|+\rho_{2}(e)\|r\|
$$

where $\rho_{1}(e)$ and $\rho_{2}(e)$ are known positive bounding functions. The above bound will be exploited to obtain the stability result presented in the next section. 


\section{Stability Analysis}

The combination of error systems in (30) and (38) yields the following stability result for the observation error and the position tracking error.

Theorem 1: The velocity observer in (14) and the control law in (17) ensure that the closed-loop observer-controller couple is semi-globally asymptotically stable in the sense that

$$
\|e(t)\|,\|\dot{\tilde{q}}(t)\| \rightarrow 0 \text { as } t \rightarrow+\infty
$$

provided that the controller and observer gains are selected to satisfy (29), (34), and controller gain $K_{c}$ and observer gain $K_{2}$ are chosen to satisfy the following constraints

$$
\begin{aligned}
& K_{c}=1+\rho_{2}+k_{n} \rho_{1}^{2} \\
& K_{2}=\alpha\left(1+\rho_{o 4}+k_{n}\left(\rho_{o 1}^{2}+\rho_{o 2}^{2}+\rho_{o 3}^{2}\right)\right)
\end{aligned}
$$

where $\rho_{1}(e), \rho_{2}(e)$ were defined in $(40), \rho_{o i}, i=1,2,3,4$ were defined in (27) and $k_{n} \in \mathbb{R}$ being a nonlinear damping gain selected to satisfy the following condition

$$
k_{n}>\left(1+\frac{\lambda_{2}}{\lambda_{1}}\|z(0)\|^{2}\right) / 2
$$

and $z(t) \in \mathbb{R}^{(3 n+p+1) \times 1}$ defined as follows

$$
z(t) \triangleq\left[\begin{array}{lllll}
s^{T} & \sqrt{P_{0}} & r^{T} & e^{T} & \tilde{\theta}^{T}
\end{array}\right]^{T} .
$$

and the positive bounding constants $\lambda_{1}, \lambda_{2} \in \mathbb{R}$ are defined as

$$
\begin{gathered}
\lambda_{1}=\frac{1}{2} \min \left\{1, m_{1}, \lambda_{\min }\left\{K_{p}\right\}\right\} \\
\lambda_{2}=\frac{1}{2} \max \left\{1, m_{2}, \lambda_{\max }\left\{K_{p}\right\}\right\} .
\end{gathered}
$$

Proof: We start our proof by introducing the following non-negative function

$$
V=V_{0}+\frac{1}{2} r^{T} M(q) r+\frac{1}{2} e^{T} K_{p} e+\frac{1}{2} \tilde{\theta}^{T} \Gamma^{-1} \tilde{\theta} .
$$

From (46), $V(t)$ can be upper and lower bounded as

$$
\lambda_{1}\|x\|^{2} \leq \lambda_{1}\|z\|^{2} \leq V \leq \lambda_{2}\|z\|^{2}
$$

where $x(t) \in \mathbb{R}^{3 n}$ is defined as

$$
x(t) \triangleq\left[\begin{array}{lll}
s^{T} & r^{T} & e^{T}
\end{array}\right]^{T} .
$$

Taking the time derivative of (46), and then substituting (12), time derivative of (13), (21), (35) and (38), and cancelling common terms results in

$$
\dot{V}=s^{T}\left[-\frac{K_{2}}{\alpha} s+N_{b}\right]+r^{T}\left[\chi-K_{c} r\right]-\alpha K_{p}\|e\|^{2}
$$

where (4) has been utilized. After applying (27) and (39) to (49), we can form the following upper bound for $\dot{V}(t)$

$$
\begin{aligned}
\dot{V} \leq & -\|e\|^{2}-\|r\|^{2}-\|s\|^{2} \\
& +\left[\rho_{o 1}\|e\|\|s\|-k_{n} \rho_{o 1}^{2}\|s\|^{2}\right] \\
& +\left[\rho_{o 2}\|r\|\|s\|-k_{n} \rho_{o 2}^{2}\|s\|^{2}\right] \\
& +\left[\rho_{o 3}\|r\|^{2}\|s\|-k_{n}^{2} \rho_{o 3}^{2}\|s\|^{2}\right] \\
& +\left[\rho_{1}\|e\|\|r\|-k_{n} \rho_{1}^{2}\|r\|^{2}\right] .
\end{aligned}
$$

After completing the squares for the terms in the brackets, we can obtain

$\dot{V} \leq-\left[1-\frac{1}{2 k_{n}}\right]\|e\|^{2}-\left[1-\frac{1}{4 k_{n}}-\frac{1}{4 k_{n}}\|r\|^{2}\right]\|r\|^{2}-\|s\|^{2}$

which using the definition of $x(t)$ in (48) can be further upper bounded as

$$
\dot{V} \leq-\left[1-\frac{1}{2 k_{n}}\left(1+\|x\|^{2}\right)\right]\|x\|^{2} .
$$

The sign of the upper bound of $\dot{V}(t)$ is determined by the term in the brackets of (52). This term has to be positive to ensure the negative semi-definiteness of $\dot{V}(t)$, that is, to ensure the negative semi-definiteness of $\dot{V}(t)$, we must have

$$
1-\frac{1}{2 k_{n}}\left(1+\|x\|^{2}\right)>0 .
$$

From (47), a sufficient condition on (53) can be obtained as

$$
1-\frac{1}{2 k_{n}}\left(1+\frac{V(t)}{\lambda_{1}}\right)>0
$$

and hence at this point the analysis can be reformulated as

$$
\dot{V} \leq-\beta\|x\|^{2} \text { provided that } 2 k_{n}>\left(1+\frac{V(t)}{\lambda_{1}}\right)
$$

where $\beta \in \mathbb{R}$ is some positive constant $(0<\beta \leq 1)$. Due to the negative semi-defineteness of $\dot{V}(t)$, the maximum value that $V(t)$ can have is its initial value, $V(0)$, therefore, from (47), a more conservative condition on $k_{n}$ can be obtained to have the following form

$$
\dot{V} \leq-\beta\|x\|^{2} \text { provided that } 2 k_{n}>1+\frac{\lambda_{2}}{\lambda_{1}}\|z(0)\|^{2}
$$

that is when $k_{n}$ is selected to satisfy (43), we can ensure that $V(t)$ is bounded, therefore, $z(t) \in \mathcal{L}_{\infty}$ (i.e., $e(t)$, $\left.r(t), s(t), P_{0}(t) \in \mathcal{L}_{\infty}\right)$. After utilizing standard signal chasing arguments, we can show that all signals in the closedloop system are bounded and $e(t)$ and $\dot{\tilde{q}}(t)$ are uniformly continuous signals (from the boundedness of their derivatives over time), furthermore, from the integration of both sides of (55), it is easy to see that $x(t) \in \mathcal{L}_{2}$ and therefore $e(t)$, $\dot{\tilde{q}}(t) \in \mathcal{L}_{2}$. Finally, after utilizing a direct application of Barbalat's Lemma [16], we can obtain the result given in (41) provided that the gain condition of (43) is satisfied.

\section{Simulation Results}

The observer based adaptive output feedback controller proposed in this paper was simulated on a two-link, directdrive, planar robot manipulator having the following dynamic model [17]

$$
\begin{aligned}
& {\left[\begin{array}{cc}
p_{1}+2 p_{3} c_{2} & p_{2}+p_{3} c_{2} \\
p_{2}+p_{3} c_{2} & p_{2}
\end{array}\right]\left[\begin{array}{c}
\ddot{q}_{1} \\
\ddot{q}_{2}
\end{array}\right.} \\
& +\left[\begin{array}{cc}
-p_{3} s_{2} \dot{q}_{2} & -p_{3} s_{2}\left(\dot{q}_{1}+\dot{q}_{2}\right) \\
p_{3} s_{2} \dot{q}_{1} \\
f_{d 1} & 0 \\
0 & f_{d 2}
\end{array}\right]\left[\begin{array}{c}
0 \\
\dot{q}_{1} \\
\dot{q}_{2}
\end{array}\right]=\left[\begin{array}{l}
\tau_{1} \\
\tau_{2}
\end{array}\right]\left[\begin{array}{l}
\dot{q}_{1} \\
\dot{q}_{2}
\end{array}\right]
\end{aligned}
$$


where $p_{1}=3.473\left[\mathrm{~kg}-\mathrm{m}^{2}\right], p_{2}=0.193\left[\mathrm{~kg}-\mathrm{m}^{2}\right], p_{3}=$ $0.242\left[\mathrm{~kg}-\mathrm{m}^{2}\right], f_{d 1}=5.3[\mathrm{Nm}-\mathrm{sec}], f_{d 2}=1.1[\mathrm{Nm}-\mathrm{sec}]$, $c_{2} \triangleq \cos \left(q_{2}\right)$, and $s_{2} \triangleq \sin \left(q_{2}\right)$. Based on (7) and (56), the system parameter vector $\theta$ can be constructed as

$$
\theta=\left[\begin{array}{lllll}
p_{1} & p_{2} & p_{3} & f_{d 1} & f_{d 2}
\end{array}\right]^{T} .
$$

The simulations were performed using the following desired link position trajectory

$$
\left.q_{d}(t)=\left[\begin{array}{l}
0.7 \sin (t)\left(1-\exp \left(-0.3 t^{3}\right)\right) \\
1.2 \sin (t)\left(1-\exp \left(-0.3 t^{3}\right)\right)
\end{array}\right] \text { [rad }\right]
$$

where the exponential term was included to ensure that $\dot{q}_{d}(0)=\ddot{q}_{d}(0)=\dddot{q}_{d}(0)=0$ and the observer-controller gains were selected as

$$
\begin{gathered}
\alpha=\operatorname{diag}\left\{\begin{array}{ll}
1.8 & 1.6
\end{array}\right\} \\
K_{o}=\operatorname{diag}\left\{\begin{array}{cc}
8 & 6
\end{array}\right\}, K_{1}=\operatorname{diag}\left\{\begin{array}{ll}
1.6 & 1.2
\end{array}\right\}, \\
K_{c}=\operatorname{diag}\left\{\begin{array}{lll}
1.6 & 1.4
\end{array}\right\}, K_{p}=\operatorname{diag}\left\{\begin{array}{ll}
32 & 24
\end{array}\right\}
\end{gathered}
$$

with the adaptation gains selected as

$$
\Gamma=\operatorname{diag}\{11.6,1.8,2.4,7.8,8.6\} .
$$

We note that all the above gains were tuned by trial-anderror until the best link position tracking performance was achieved. The parameter estimate $\hat{\theta}(t)$ was initialized to zero, and the simulations were performed at a sampling frequency of $2 \mathrm{kHz}$.

The results are shown in Figures 1-3. The link position tracking errors are depicted in Figure 1, while the parameter estimates and control torques are shown in Figures 2 and 3. From Figure 1, it is clear that the tracking objective was met.
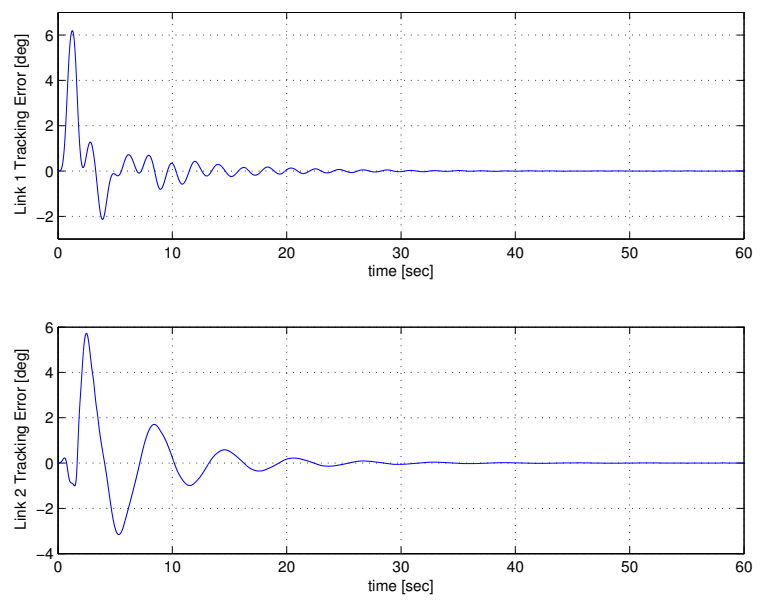

Fig. 1. Link Tracking Errors

\section{CONCLUSION}

In this paper, we have presented a new observer based adaptive output feedback tracking controller for robot manipulators. A novel observer-controller couple was introduced that ensured semi-globally asymptotic tracking despite the lack of link velocity measurements and parametric uncertainties in the system dynamics. Simulation results were presented to illustrate the tracking performance of the observercontroller couple. Future work will focus on extending the
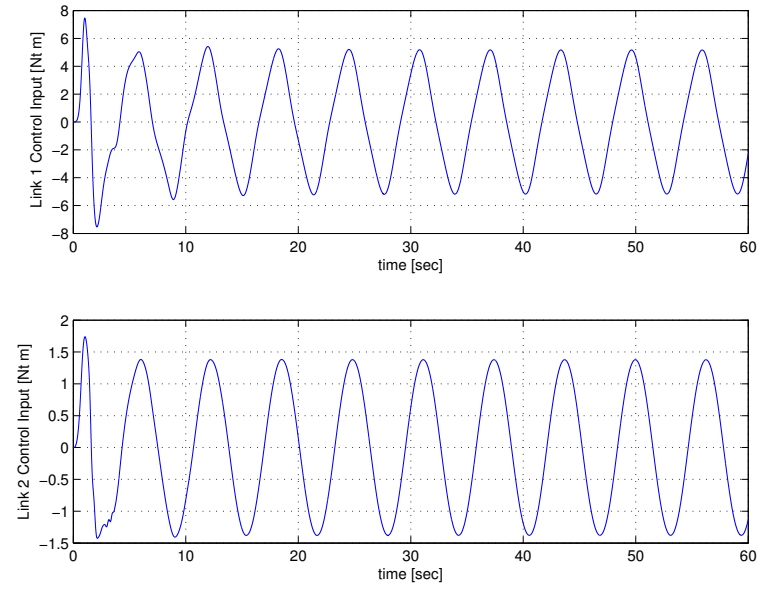

Fig. 2. Control Torque Inputs

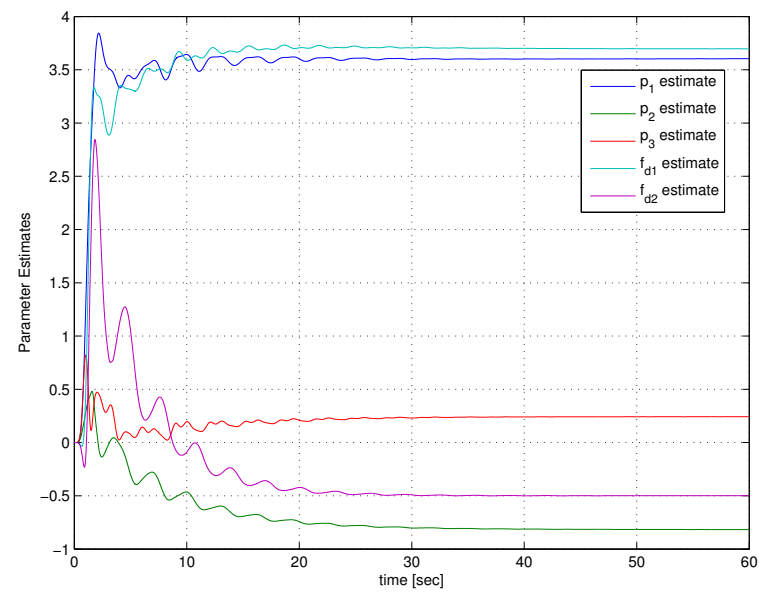

Fig. 3. Parameter Estimates

proposed result to design repetitive learning output feedback controllers for robot manipulators.

\section{REFERENCES}

[1] S. Arimoto, V. Parra-Vega, and T. Naniwa, "A Class of Linear Velocity Observers for Nonlinear Mechanical Systems," Proc. Asian Control Conf., Tokyo, Japan, pp. 633-636, 1994.

[2] H. Berghuis and H. Nijmeijer, "A Passivity Approach to ControllerObserver Design for Robots," IEEE Tr. on Robotics and Automation, vol. 9, no. 6, pp. 740-754, 1993.

[3] T. C. Burg, D. M. Dawson, and P. Vedagarbha, "A Redesigned DCAL Controller without Velocity Measurements: Theory and Demonstration," Robotica, vol. 15, pp. 337-346, 1997.

[4] E. Zergeroglu, W. E. Dixon, D. Haste, and D. M. Dawson, "A Composite Adaptive Output Feedback Tracking Controller for Robotic Manipulators," Robotica, vol. 17, pp. 591-600, 1999.

[5] J. Yuan and Y. Stepanenko, "Robust Control of Robotic Manipulators without Velocity Measurements," Int. J. Robust and Nonlinear Control, vol. 1, pp. 203-213, 1991.

[6] F. Zhang, D. M. Dawson, M. S. de Queiroz, and W. E. Dixon, "Global Adaptive Output Feedback Control of Robot Manipulators," IEEE Tr. on Automatic Control, vol. 45, no. 6, pp. 1203-1208, 2000.

[7] E. Zergeroglu, D. M. Dawson, M. S. de Queiroz, and M. Krstic, "On Global Output Feedback Control of Robot Manipulators," Proc. IEEE Int. Conf. on Decision and Control, Sydney, Australia, pp. 5073-5078, 2000.

[8] S. Nicosia and P. Tomei, "Robot Control by Using Only Position Measurements," IEEE Tr. Automatic Control, vol. 35, no. 9, pp. 1058$1061,1990$. 
[9] B. Xian, M. S. de Queiroz, D. M. Dawson, and M. L. McIntyre, "Output Feedback Variable Structure-like Control of Nonlinear Mechanical Systems," Proc. IEEE Int. Conf. on Decision and Control, Maui, HA, USA, pp. 368-373, 2003.

[10] A. Abdessameud and M. F. Khelfi, "A Variable Structure Observer for the Control of Robot Manipulators," Int. J. Appl. Math. Compt. Sci., vol. 16, no. 2, pp. 189-196, 2006.

[11] F. L. Lewis, C. T. Abdallah, and D. M. Dawson, Control of Robot Manipulators, New York, NY, USA: Macmillan Publishing Co., 1993.

[12] P. Tomei, "Adaptive PD Controller for Robot Manipulators," IEEE Tr. on Robotics and Automation, vol. 7, no. 4, pp. 565-570, 1991

[13] N. Sadegh and R. Horowitz, "Stability and Robustness Analysis of a Class of Adaptive Controllers for Robot Manipulators," Int. J. Robotics Research, vol. 9, no. 3, pp. 74-92, 1990.

[14] H. K. Khalil, Nonlinear Systems, 3rd Edition, New York, NY, USA: Prentice Hall, 2002.

[15] B. Xian, D. M. Dawson, M. S. de Queiroz, and J. Chen, "A Continuous Asymptotic Tracking Control Strategy for Uncertain Nonlinear Systems," IEEE Tr. on Automatic Control, vol. 47, no. 7, pp. 1206-1211, 2004.

[16] M. Krstic, I. Kanellakopoulos, and P. Kokotovic, Nonlinear and Adaptive Control Design, New York, NY, USA: John Wiley \& Sons, 1995.

[17] Direct Drive Manipulator Research and Development Package Operations Manual, Integrated Motion Inc., Berkeley, CA, USA, 1992.

\section{APPENDIX I}

\section{PROOF OF BOUNDS}

In this appendix, we illustrate how the upper bounds for $N_{b}(t)$ in (27) and $\chi(t)$ in (40) are obtained. We start with exploiting the expression given in (26), which can be rewritten in the following form

$$
\begin{aligned}
N_{b}= & \left(M^{-1}(q)-M^{-1}\left(q_{d}\right)\right)\left(M\left(q_{d}\right) \ddot{q}_{d}+Y_{d} \tilde{\theta}\right) \\
& +M^{-1}(q)\left\{V_{m}\left(q_{d}, \dot{q}_{d}\right) \dot{q}_{d}-V_{m}\left(q, \dot{q}_{d}\right) \dot{q}_{d}\right\} \\
& +M^{-1}(q)\left\{2 V_{m}(q, \dot{e}) \dot{q}_{d}-V_{m}(q, \dot{e}) \dot{e}\right\} \\
& +M^{-1}(q)\left\{G\left(q_{d}\right)-G(q)+F_{d}\left(\dot{q}_{d}-\dot{q}\right)\right\} \\
& +M^{-1}(q)\left\{K_{p} e+K_{c} r+K_{c} s\right\}
\end{aligned}
$$

where (5) has been utilized. After applying (3), (6), and (9), we can upper bound (60) as

$$
\begin{aligned}
N_{b} \leq & \left\{\zeta_{m 1} m_{2}\left\|\ddot{q}_{d}\right\|+\left\|Y_{d} \tilde{\theta}\right\|+\frac{1}{m_{1}} \zeta_{c 2}\left\|\dot{q}_{d}\right\|\right. \\
& \left.+\frac{1}{m_{1}} \lambda_{\max }\left\{K_{p}\right\}+\frac{1}{m_{1}} \zeta_{g}\right\}\|e\| \\
& +\left\{\frac{2}{m_{1}} \zeta_{c 1}\left\|\dot{q}_{d}\right\|+\frac{1}{m_{1}} \zeta_{f}+\frac{1}{m_{1}} \lambda_{\max }\left\{K_{c}\right\}\right\}\|r\| \\
& +\frac{1}{m_{1}} \zeta_{c 1}\|r\|^{2}+\frac{1}{m_{1}} \lambda_{\max }\left\{K_{c}\right\}\|s\|
\end{aligned}
$$

where the fact that $\|r(t)\| \geq\|\dot{e}(t)\|$ has been utilized. From the structure of (61), it is clear that the bounding function of (27) are

$$
\begin{aligned}
\rho_{01}= & \zeta_{m 1} m_{2}\left\|\ddot{q}_{d}\right\|+\frac{1}{m_{1}} \zeta_{c 2}\left\|\dot{q}_{d}\right\| \\
& +\frac{1}{m_{1}} \lambda_{\max }\left\{K_{p}\right\}+\frac{1}{m_{1}} \zeta_{g}, \\
\rho_{02}= & \frac{2}{m_{1}} \zeta_{c 1}\left\|\dot{q}_{d}\right\|+\frac{1}{m_{1}} \zeta_{f}+\frac{1}{m_{1}} \lambda_{\max }\left\{K_{c}\right\}, \\
\rho_{03}= & \frac{1}{m_{1}} \zeta_{c 1}, \quad \text { and } \quad \rho_{04}=\frac{1}{m_{1}} \lambda_{\max }\left\{K_{c}\right\}
\end{aligned}
$$

and the bound given in (27) is valid.$$
\text { the bound given in (27) is valid. }
$$

For the expression in (40), we start with the previously found upper bound on the same term [11] (see Chapter 6 equation 6.2-9) as

$$
\|\chi\| \leq \zeta_{1}\|e\|+\zeta_{2}\|e\|^{2}+\zeta_{3}\|r\|+\zeta_{4}\|r\|\|e\|
$$

where $\zeta_{i}, i=1,2,3,4$ are positive bounding constants that depend on the desired trajectory and physical parameters (i.e., link mass, link length, friction coefficients, etc.). The right-hand-side of (63) can be written to have the following form

$$
\|\chi\| \leq\left(\zeta_{1}+\zeta_{2}\|e\|\right)\|e\|+\left(\zeta_{3}+\zeta_{4}\|e\|\right)\|r\|
$$

from which it is quite obvious that when the bounding functions $\rho_{1}(e)$, and $\rho_{2}(e)$ are selected as

$$
\begin{aligned}
& \rho_{1}(e)=\zeta_{1}+\zeta_{2}\|e\| \\
& \rho_{2}(e)=\zeta_{3}+\zeta_{4}\|e\|
\end{aligned}
$$

bound given in (40) is satisfied. 